\title{
Erratum to: Biomechanical and histological evaluation of four different titanium implant surface modifications: an experimental study in the rabbit tibia
}

\author{
José Luis Calvo-Guirado ${ }^{1}$ - Marta Satorres ${ }^{1} \cdot$ Bruno Negri $^{1}$ • \\ Piedad Ramirez-Fernandez ${ }^{1}$. Jose Eduardo Maté-Sánchez de Val ${ }^{1}$. \\ Rafael Delgado-Ruiz ${ }^{2}$ Gerardo Gomez-Moreno ${ }^{3}$ - Marcus Abboud ${ }^{2}$. \\ Georgios E. Romanos ${ }^{2}$
}

Published online: 20 June 2015

(C) Springer-Verlag Berlin Heidelberg 2015

Erratum to: Clin Oral Invest 18 (5): 1495-1505

DOI 10.1007/s00784-013-1120-2

The original version of the above article contained a mistake in the name of one of the authors. Jose Eduardo Maté-Sánchez should be captured as Jose Eduardo Maté-Sánchez de Val.

Georgios E. Romanos

georgios.romanos@stonybrookmedicine.edu

1 Faculty of Medicine and Dentistry, University of Murcia, Murcia, Spain

2 Stony Brook University, Stony Brook, NY, USA

3 Faculty of Dentistry, University of Granada, Granada, Spain 